\title{
The link between weight shift asymmetry and gait disturbances in chronic hemiparetic stroke patients
}

This article was published in the following Dove Press journal:

Clinical Interventions in Aging

\author{
Andrzej Szopa' \\ Małgorzata Domagalska- \\ Szopa ${ }^{2}$ \\ Anetta Lasek-Bal ${ }^{3}$ \\ Amadeusz $\dot{Z}_{a k}{ }^{3}$ \\ 'Department of Physiotherapy, \\ 2Department of Medical \\ Rehabilitation, School of Health \\ Sciences in Katowice, ${ }^{3}$ Department \\ of Neurology, Professor Leszek \\ Giec Upper Silesian Medical Centre, \\ Medical University of Silesia, \\ Katowice, Poland
}

Introduction: While the asymmetry of body posture and the asymmetrical nature of hemiparetic gait in poststroke (PS) patients are well documented, the role of weight shift asymmetry in gait disorders after stroke remains unclear.

Objective: We examined the association of weight-bearing asymmetry (WBA) between paretic and nonparetic lower limbs during quiet standing with the degree of deviation of hemiplegic gait from normal gait evaluated by the Gillette Gait Index (GGI) incorporating 16 distinct clinically important kinematic and temporal parameters in chronic PS patients.

Participants and methods: Twenty-two ambulatory patients with chronic stroke aged between 50 and 75 years were included in this study. Fourteen patients had hemiparesis on the nondominant side and 8 on the dominant side. The mean time PS was 2 years and 6 months. The reference group consisted of 22 students from the University of the Third Age presenting no neurological disorders. The examination consisted of posturographic weight-bearing (WB) distribution and 3-dimensional gait analyses.

Results: A significant positive relationship between WBA and GGI was revealed. Moreover, we observed a significant negative association between WBA and paretic step length and walking speed. With regard to kinematic data, the range of motion of knee flexion and peak dorsiflexion in the swing phase of the paretic leg were significantly negatively associated with WBA.

Conclusion: Although further research is needed to determine a causal link between postural control asymmetry and gait disturbance in hemiplegics, our findings support the inclusion of WB measurements between paretic and nonparetic body sides in early assessment after stroke.

Keywords: stroke, weight-bearing, 3DGA, gait disturbances, Gillette Gait Index

\section{Introduction}

Damage to the central nervous system (CNS) caused by stroke is a major reason underlying motor and cognitive function impairment. ${ }^{1}$ The functional problems encountered by poststroke (PS) patients are heterogeneous and mainly comprise impaired postural control and abnormal gait pattern with marked asymmetry. ${ }^{2-4}$

Quiet standing postures of PS are characterized by the following: 1) weight-bearing asymmetry (WBA) with a shift in the mean position of the center of pressure (COP) toward the nonparetic side ${ }^{5}{ }^{2}$ ) increase in COP displacement, which reflects larger postural sway; and 3) a small area of stability and asymmetrical contribution of lower limbs to balance control compared with age-matched healthy controls. ${ }^{6-10}$

Although a few studies have documented the successful recovery of standing postural control in hemiplegics after stroke, the persistent presence of these 3 main patterns generally characterizes the static standing posture of hemiparetic patients. $^{9-11}$
Correspondence: Małgorzata

Domagalska-Szopa

School of Health Sciences in Katowice, Medical University of Silesia, Medyków

12, 40-752 Katowice, Poland

$\mathrm{Tel}+48601516725$

Email mdomagalska@sum.edu.pl (c)
hereby accept the Terms. Non-commercial uses of the work are permitted without any further permission from Dove Medical Press Limited, provided the work is properly attributed. For permission hereby accept the Terms. Non-commercial uses of the work are permitted without any further permission from Dove Medcal
for commercial use of this work, please see paragraphs 4.2 and 5 of our Terms (https://www.dovepress.com/terms.php). 
Improvements in motor activities, including walking, following stroke are variable, with $65 \%-85 \%$ of patients regaining the ability to walk independently within 6 months after brain stroke. However, deviations in gait are commonly present. ${ }^{12}$

Normal gait tends to be symmetrical, both spatially and temporally, with slight interlimb differences, while PS gait is generally asymmetrical. ${ }^{411-14}$ The hemiplegic gait pattern is characterized by spatial and temporal differences between the paretic and nonparetic lower limb and biomechanical (kinematic, kinetic, and muscular activity) parameter asymmetries. Several studies have reported temporal asymmetry in stance time, single stance time, double support time, and swing time ${ }^{15-17}$ and spatial asymmetry that is mainly concerned with step length in individuals PS. ${ }^{14,17}$ In addition to the asymmetry in spatial and temporal parameters, hemiplegics after stroke present reduced walking speed relative to healthy subjects. ${ }^{14,18}$

Although spatiotemporal parameters are the most commonly used variables for differentiation between hemiplegic and normal gaits from the onset, recent studies have additionally focused on biomechanical differences. In PS gait, a range of kinematic deviations has been reported. ${ }^{12,13}$ As determined from 3-dimensional gait analysis (3DGA), joint kinematics of hemiplegic patients differ from those of healthy individuals in both the stance and swing phases of gait. In addition, these patients exhibit considerable interindividual variabilities. ${ }^{19}$ The most common kinematic deviations involve sagittal plane disturbances of hip, knee, and ankle motions in the hemiplegic limb, including limited or reduced hip/knee flexion and reduced ankle dorsiflexion or continuous plantar flexion.

In recent years, many studies have focused on the associations among gait disturbance, sensorimotor deficits, biomechanical impairment, energy expenditure, and frequency of falls. ${ }^{3,5,10,12}$ Studies aimed at better understanding of gait asymmetry following stroke have explored the relationship between the asymmetry of standing balance control and walking PS. The results of these studies suggest that WBA and decreased contribution of the paretic lower limb to the control of quiet standing contribute to gait asymmetry PS. ${ }^{20-22}$ 3DGA research to date has solely focused on spatiotemporal PS gait parameters. WBA and decreased contribution of the paretic limb to the control of quiet standing have been shown to be related to increased asymmetry of swing time, stance time, and, to a lesser extent, step length. ${ }^{20}$ However, the overall evidence for associations between WBA and complex PS gait deviation is insufficient for conclusive interpretation.

Although quantitative 3DGA is an excellent indicator of gait dysfunction in hemiparetic patients, the method results in the generation of a considerable amount of data that requires complex interpretation. To overcome these problems, an index for quantifying deviations from normal gait, Gillette Gait Index (GGI), previously known as the normalcy index, ${ }^{23}$ was introduced. GGI is a multivariate index combining 16 gait variables (including temporal, spatial, and kinematic parameters) to derive a single measure of overall gait function. This index estimates the deviation of a patient's gait from the normal pattern and has been validated in several patient populations, including PS individuals. ${ }^{24}$

The present study aimed at verifying the hypothesis that the degree of deviation of hemiparetic from normal gait patterns evaluated based on GGI depends on the degree of WBA during quiet standing between paretic and nonparetic body sides in PS patients. The additional purpose of this study was to evaluate potential correlations of WBA during quiet standing with the 16 distinct gait parameters constituting GGI.

\section{Participants and methods}

Approval for this study was obtained from the Research Ethics Committee of the Medical University of Silesia (Poland). Each patient provided written informed consent prior to enrolment.

Individuals with chronic hemiparetic stroke were recruited from outpatients of local rehabilitation centers (from January to December 2016) and voluntarily consented to participate in the study. The inclusion criteria were as follows: 1) a single onset of ischemic stroke at least a year earlier; 2) ability to stand independently for 30 seconds without support; 3) ability to walk independently without assistance or devices for at least $10 \mathrm{~m}$; and 4) ability to follow verbal requests. The exclusion criteria included musculoskeletal impairments affecting gait kinematics and neurological conditions other than unilateral stroke (eg, Parkinson's disease) or medical contraindications to performing treadmill walking. The following information was extracted from individual clinical documents of patients: age, sex, type of stroke, date of stroke, and paretic side.

Twenty-two participants who met the above inclusion criteria were included in the study. The experimental group consisted of 14 male and 8 female subjects with a mean age of 68 years and 2 months (50-75 years). In total, 14 patients had hemiparesis on the nondominant side and 8 on the dominant side. The mean time interval (and SD) between the onset of stroke and return to independent walking was $10.8 \pm 6.0$ weeks (7.3-27.6 weeks). Mean time PS was 2 years and 6 months $(\mathrm{SD}=1.92)$.

Each patient with stroke was subjected to clinical examination, including motor recovery assessment, muscle tone assessment, and muscle strength evaluation of the 4 muscle 
groups of the paretic lower limbs (knee flexors and extensors and ankle dorsi- and plantar-flexors). Motor recovery of patients was assessed clinically by using Brunnström recovery stages based on the degree of spasticity and appearance of voluntary movement. ${ }^{25}$ Muscle tone was rated by using a 5-point Modified Modified Ashworth Scale (MMAS). ${ }^{26}$ Overall MMAS scores were calculated as a mean of the individual MMAS scores of the above 4 muscle groups of the paretic lower limb. Each muscle group was graded from 0 to 4 . The total MMAS score ranged from 0 (normal muscle tone) and 1 (slight increase in muscle tone) to 4 (severe spasticity). Muscle strength was assessed by using the Medical Research Council (MRC) scale. ${ }^{27}$ The total MCR score was calculated as a mean of MRC scores of the above 4 muscle groups in the paretic lower limb. Each muscle group was graded from 0 to 5 , with total MRC score ranging from 0 (total paralysis) to 5 (normal strength). ${ }^{27}$ Table 1 presents the participant characteristics, including recovery stages, sensorimotor deficits, and biomechanical impairments.

The reference group consisted of 22 age-matched healthy students from the University of the Third Age, including 10 male and 10 female subjects with a mean age of 59 years and 3 months ( $S D=10.1$; age range $=61-75$ years). Subjects in this group had no neurological disorders, prior neurosurgical treatment, significant visual defects, past injuries of lower limbs, acute pain incidence up to 3 months before examination, or exposure to pharmacological treatment agents, such as hypnotics, antidepressants, and sedatives.

\section{Methods}

The examination included two interrelated parts: 1) posturographic weight-bearing (WB) distribution and 2) 3DGA.

\section{Posturographic WB distribution}

During WB distribution measurement, the subjects stood comfortably with open eyes in a quiet stance position. The distance between their parallel heels was $\sim 3 \mathrm{~cm}$. The WB distribution was measured by using a force platform (pressure distribution measurement [PDM]; Zebris Medizintechnik GmbH, Isny im Allgäu, Germany) with FootPrint software. The measurement was recorded 3 times ( 3 trials, each lasting for 30 seconds, with a 30 -second pause between trials, in 15th second of each trial). The WB between paretic and nonparetic body sides in the experimental group and between dominant and nondominant sides in the control group was registered. The mean values from 3 trials were used for future analysis.

\section{DGA}

For the 3DGA, we used the Compact Measuring System for $3 \mathrm{D}$ real-time motion analysis based on 15 active ultrasonic markers (5 triplicate ultrasound markers) with WinGait software (Zebris Medizintechnik $\mathrm{GmbH}$ ). The gait data were recorded as the subjects walked without shoes and without assistive devices on a treadmill (Alfa XL; Kettler $\mathrm{GmbH}$, Ense, Germany). Typical overground walking speed (spontaneous) of and time taken to walk $10 \mathrm{~m}$ by each subject were collected before the gait analysis. Based on the spontaneous speed of walking, treadmill belt speeds were calculated as values in kilometers per hour. Before data collection, the participants walked on the treadmill for 3 minutes to familiarize themselves with treadmill walking. Treadmill walking speed was reduced if the participant felt that the speed was not a comfortable walking speed. Recording of kinematic signals was conducted during 10 seconds at each of the next

Table I Demographic and clinical characteristics

\begin{tabular}{llll}
\hline Characteristics & $\begin{array}{l}\text { Poststroke group } \\
(\mathbf{n = 2 2})\end{array}$ & $\begin{array}{l}\text { Control group } \\
(\mathbf{n = 2 2})\end{array}$ & $\begin{array}{l}\text { Statistical tests; } \\
\mathbf{P} \text {-values }\end{array}$ \\
\hline Age (years), mean (SD) & $68(\mathrm{II})$ & $69(7)$ & $t=1.22 ; P=0.75$ \\
Gender (men), $\mathrm{n}(\%)$ & $14(64)$ & $14(64)$ & $\chi^{2}=0.0 ; P=1.0$ \\
$\mathrm{Al}^{\mathrm{a}}$, mean (SD) & $0.09(0.06)$ & $0.03(0.01)$ & $t=3.07 ; P=0.00$ \\
GGI, mean (SD) & $86.8(45.2)$ & $13.9(6.3)$ & $t=6.86 ; P=0.00$ \\
Walking speed (m/s), mean (SD) & $0.41(0.09)$ & $1.39(0.23)$ & $t=4.67 ; P=0.00$ \\
Step length asymmetry (m), mean (SD) & $0.19(0.02)$ & $0.08(0.01)$ & $t=4.03 ; P=0.00$ \\
Time since stroke (months), mean (SD) & $30(1.92)$ & & \\
Brunnström recovery stage, maximum 6 (grade range) & $4-6$ & & \\
Modified Modified Ashworth Scale, maximum 4 (grade range) & $0-3$ & & \\
Medical Research Council Scale, minimum 0 (grade range) & $3-5$ & & \\
\hline
\end{tabular}

Notes: Asymmetry of WB distributions, the GGI variables and selected spatiotemporal gait parameters (step length and walking speed) for the poststroke group ( $\mathrm{n}=22$ ) and control group $(n=22)$, means $(S D)$ of the outcome $(95 \% \mathrm{Cl})$, and comparison between the groups (statistical tests and $P$-values). ${ }^{a} A l=(N P-P) /(N P+P) ; N P=$ the nonparetic/ dominant side percentage load distribution; and $\mathrm{P}=$ paretic/nondominant side percentage load distribution. ${ }^{b} \mathrm{NP}-\mathrm{P} ; \mathrm{NP}=$ the step length of nonparetic/dominant lower limb; and $\mathrm{P}=$ the step length of paretic/nondominant lower limb.

Abbreviations: Al, asymmetry index; GGI, Gillette Gait Index; WB, weight-bearing. 
minute (from 4th through 10th minute) of treadmill walking. For each subject, from 8 to 10 gait cycles were recorded. An experienced physical therapist selected the 6 gait cycles per subject for further analysis, which were the most characteristic of the subject in his opinion. Separate measurements for the nonparetic and paretic gait cycles for each subject were obtained. The kinematic data were averaged from 3 randomly selected cycles of these 6 .

To characterize the gait pattern, the GGI was used, according to the procedure described by Schutte et al. ${ }^{23}$ The GGI was calculated separately for paretic and nonparetic lower limbs based on the 16 selected gait parameters taken from the objective 3DGA data, including 1) stance phase, expressed as the percentage of the gait cycle; 2) walking speed, normalized to leg length; 3) cadence; 4) mean pelvic tilt; 5) range of motion (ROM) of pelvic tilt; 6) mean pelvic rotation; 7) minimum hip flexion; 8) ROM of hip flexion/ extension; 9) peak hip abduction in swing; 10) mean hip rotation in stance; 11) knee flexion at initial contact; 12) time to peak knee flexion in swing, expressed as the percentage of the gait cycle; 13) ROM of knee flexion; 14) peak dorsiflexion in stance; 15) peak dorsiflexion in swing; and 16) mean foot progression. ${ }^{23}$

In order to determine the intraobserver agreement, the intraclass correlation coefficient $(\mathrm{ICC}=2.1)$ with $95 \% \mathrm{CI}$ for the gait analysis (GGI for paretic lower limb) based on two examinations performed by the same assessor of 10 subjects was calculated. GGI for paretic lower limb demonstrated good to high level of intraobserver repeatability, with the ICC ranging from 0.68 to 0.83 . The repeatability was assessed based on the interpretation of ICC (similar to the interpretation of the correlation coefficient): $0-0.2$, very low; 0.21-0.4, low; 0.41-0.6, medium; 0.61-0.8, good; and $0.8-1$, high repeatability. ${ }^{28}$

\section{Data analysis}

The normality of the distribution of the analyzed parameters was assessed by using the skewness and kurtosis measures and the Shapiro-Wilk test. Descriptive statistics were calculated for the clinical characteristics of both experimental and control groups. Normally distributed variables are summarized as the means and SDs, and non-normally distributed variables are presented as the median and range.

Asymmetry index (AI) of the posturographic WB distribution between body sides was calculated as follows: $\mathrm{AI}=\frac{\mathrm{NP}-\mathrm{P}}{\mathrm{NP}+\mathrm{P}} ;$ where $\mathrm{NP}=$ the nonparetic/dominant side percentage load distribution, and $\mathrm{P}=$ paretic/nondominant side percentage load distribution. The AI, based on the difference between weight body sides, is a simple way to quantify WB. AI of 0 represents perfect symmetry of WB. ${ }^{29}$ The step length asymmetry was calculated as the difference between nonparetic and paretic step lengths of PS participants and between nondominant and dominant ones of controls.

To investigate differences in gait patterns, means from the healthy and stroke groups were compared on GGI and specific 16 gait parameters that composed the GGI for the paretic leg of stroke participants and nondominant one of controls using unpaired $t$-tests. Nonparametric statistics (Mann-Whitney $U$-test) were also used, when the data failed assumptions of normality. To better understand the effect of stroke on gait, the differences between the groups were provided as means and 95\% CI. This type of analysis was chosen because, while the null hypothesis, significance tests use probability levels (eg, $P<0.5$ ).

The Spearman's rank correlation test was used to examine the relationship between the posturographic WB distribution and GGI as well as the specific 16 gait parameters that composed the GGI. The correlations were performed only for the tested group. Coefficients with a $P$-value $<0.05$ were considered significant. The correlations were interpreted according to the guidelines adopted from Altman: ${ }^{28} 0-0.2$, poor; 0.21-0.4, fair; 0.41-0.6, moderate; 0.61-0.8, good; and $0.81-1$, very good. The software package Statistica (Version 12.0) was used for statistical analysis.

\section{Results}

Table 1 summarizes the mean (SD) values for demographics, asymmetry of WB distributions, GGI variables, and selected spatiotemporal gait parameters (step length and walking speed) for the healthy and stroke groups. We observed no marked differences between the groups in terms of age $(P=0.80)$ or gender $(P=1.00)$. Notably, the values for AI $(P=0.00)$, GGI $(P=0.00)$, walking speed $(P=0.00)$, and asymmetry of step length $(P=0.00)$ were significantly different between healthy and stroke participants.

Table 2 presents the descriptive statistics for the 16 distinct gait data sets constituting GGI of paretic lower limb of stroke participants relative to the nondominant lower limb of controls. The paretic lower limb displayed significant differences from the nondominant limb in terms of walking speed and cadence (both $P=0.00$ ), mean ROM of pelvic tilt and mean pelvic rotation (all $P=0.00$ ), ROM of hip and knee flexion (both $P=0.00$ ), hip abduction in the swing phase $(P=0.00)$, and peak dorsiflexion in both stance and swing phases (both $P=0.00$ ). The paretic and 
Table 2 The characteristics of gait parameters

\begin{tabular}{|c|c|c|c|}
\hline Outcome & $\begin{array}{l}\text { Poststroke group } \\
(n=22)\end{array}$ & $\begin{array}{l}\text { Control group } \\
(n=22)\end{array}$ & $\begin{array}{l}\text { Statistical tests; } \\
P \text {-values }\end{array}$ \\
\hline I. Time of toe-off (\% gait cycle), mean (SD) & $59.5(6.78)$ & $57.7(9.38)$ & $t=0.75 ; P=0.45$ \\
\hline 2. Walking speed/leg length (m/s), mean (SD) & $0.56(0.03)$ & $1.27(0.33)$ & $t=-9.81 ; P=0.00$ \\
\hline 3. Cadence (step/second), mean (SD) & $0.73(0.4 \mathrm{I})$ & $0.92(0.12)$ & $t=-4.54 ; P=0.00$ \\
\hline 4. Mean pelvic tilt $\left({ }^{\circ}\right)$, mean $(S D)$ & $13.67(3.12)$ & $6.28(5.78)$ & $t=5.27 ; P=0.00$ \\
\hline 5. ROM of pelvic tilt $\left({ }^{\circ}\right)$, mean (SD) & $8.24(3.21)$ & $4.56(1.42)$ & $t=4.90 ; P=0.00$ \\
\hline 6. Mean pelvic rotation $\left({ }^{\circ}\right)$, mean $(\mathrm{SD})$ & $-8.6 \mathrm{I}(6.75)$ & $0.4 \mathrm{I}(2.96)$ & $t=-5.74 ; P=0.00$ \\
\hline 7. Minimum hip flexion $\left({ }^{\circ}\right)$, median (range) & $-12.36([-37.02]-18.20)$ & $-10.48([-18.39]-[-2.96])$ & $U=210 ; P=0.45$ \\
\hline 8. ROM of hip flexion/extension $\left({ }^{\circ}\right)$, mean (SD) & I4.37 (3.5I) & $32.36(6.06)$ & $t=-12.03 ; P=0.00$ \\
\hline 9. Peak hip abduction in swing $\left({ }^{\circ}\right)$, mean (SD) & II.54 (5.76) & $1.76(4.23)$ & $t=6.4 \mathrm{I} ; P=0.00$ \\
\hline 10. Mean hip rotation in stance $\left({ }^{\circ}\right)$, median (range) & $15.42(10.44-27.40)$ & $7.39(-8.20-17.70)$ & $U=48.00 ; P=0.00$ \\
\hline II. Knee flexion at IC $\left(^{\circ}\right)$, mean $(S D)$ & $6.07(3.01)$ & $6.93(3.36)$ & $t=-0.89 ; P=0.37$ \\
\hline 12. Time of peak knee flex in swing (\%), mean (SD) & $44.94(12.55)$ & $62.96(7.52)$ & $t=-0.77 ; P=0.45$ \\
\hline 13. ROM of knee flexion $\left({ }^{\circ}\right)$, mean (SD) & $30.23(5.79)$ & $58.70(5.20)$ & $t=-15.32 ; P=0.00$ \\
\hline 14. Peak dorsiflexion in stance $\left({ }^{\circ}\right)$, mean (SD) & $-1.67(1.94)$ & $13.76(3.52)$ & $t=-18.01 ; P=0.00$ \\
\hline 15. Peak dorsiflexion in swing $\left({ }^{\circ}\right)$, mean (SD) & $-2.75(2.38)$ & $6.77(3.78)$ & $t=-9.98 ; P=0.00$ \\
\hline 16. Mean foot progression $\left({ }^{\circ}\right)$, median (range) & $-0.93(-30.00-24.00)$ & 13.26 (8.69-20.18) & $U=32 ; P=0.00$ \\
\hline
\end{tabular}

Note: Means (SD) of the 16 gait parameters that composed the GGI for the paretic lower limb (poststroke group; $\mathrm{n}=22$ ) and nondominant lower limb (control group; $\mathrm{n}=22$ ), means $(95 \% \mathrm{Cl})$ of the between-group differences and statistical tests and $P$-values.

Abbreviations: GGI, Gillette Gait Index; IC, initial contact; ROM, range of motion; $t$, Student's $t$-test for independent samples; $U$, Mann-Whitney $U$-test.

nondominant values for time of toe-off $(P=0.45)$, minimum hip flexion and rotation (both $P=0.45$ ), and knee flexion at initial contact and time of peak knee flex in the swing phase ( $P=0.37$ and $P=0.45$, respectively) of the stroke group were not significantly different from those of the control group (Table 2).

Table 3 summarizes the significant correlation coefficients (significance at $P$-values $<0.05$ ) of WBA with GGI and step length asymmetry in addition to the 16 individual gait parameters constituting GGI in the paretic lower limbs of the experimental groups.

Although correlations were only moderate $(0.41-0.60)$ or good (0.61-0.8), the significant positive relationship between WBA and GGI supported our main hypothesis. Moreover, we observed a significant negative relationship between WBA and both spatiotemporal parameters, walking speed and paretic step length. Among the kinematic gait parameters, only ROM of knee flexion and peak dorsiflexion in the swing phase of the paretic lower limb were significantly negatively associated with WBA.

\section{Discussion}

Overall, PS participants in this study presented asymmetry during standing and walking relative to healthy individuals.

The WBA in quiet standing in PS group was significantly larger than that in the control group. The pattern of WBA was characteristically based on more weight on the nonparetic than on the paretic limb and was similar to that reported in previous studies. ${ }^{7,20,30,31}$ While the difference between nonparetic and paretic body sides presents a simple means to quantify asymmetry, for WB and gait parameters in the PS population, asymmetry may be effectively quantified by calculating symmetry

Table 3 The correlations between asymmetry index and Gillette Gait Index

\begin{tabular}{lll}
\hline Parameter & Al & \\
\cline { 2 - 3 } & $\boldsymbol{r}$ & P-value \\
\hline I. Time of toe-off (\% gait cycle) & - & - \\
2. Walking speed/leg length $(\mathrm{km} / \mathrm{h})$ & $-0.5 \mathrm{I}$ & $0.0 \mathrm{I}$ \\
3. Cadence $($ step/second) & - & - \\
4. Mean pelvic tilt $\left({ }^{\circ}\right)$ & - & - \\
5. ROM of pelvic tilt $\left({ }^{\circ}\right)$ & - & - \\
6. Mean pelvic rotation $\left({ }^{\circ}\right)$ & - & - \\
7. Minimum hip flexion $\left({ }^{\circ}\right)$ & - & - \\
8. ROM of hip flexion/extension $\left({ }^{\circ}\right)$ & - & - \\
9. Peak hip abduction in swing $\left(^{\circ}\right)$ & - & - \\
I0. Mean hip rotation in stance $\left({ }^{\circ}\right)$ & - & - \\
II. Knee flexion at IC $\left({ }^{\circ}\right)$ & - & - \\
I2. Time of peak knee flex in swing $(\%)$ & - & - \\
13. ROM of knee flexion $\left({ }^{\circ}\right)$ & -0.48 & 0.04 \\
14. Peak dorsiflexion in stance $\left({ }^{\circ}\right)$ & - & - \\
I5. Peak dorsiflexion in swing $\left({ }^{\circ}\right)$ & -0.61 & 0.00 \\
I6. Mean foot progression $\left({ }^{\circ}\right)$ & - & - \\
GGI & 0.47 & 0.03 \\
Step length (m) & -0.68 & 0.00 \\
\hline
\end{tabular}

Notes: Statistically significant correlations between Al and GGI, step length, and 16 distinct gait parameters that composed the GGI for the paretic lower limbs for the poststroke participants. $r$, Spearman's rank correlation; $P$-value, statistical significance. Al of the posturographic weight-bearing distribution between body sides was calculated as follows: NP = the nonparetic/dominant side percentage load distribution, and $\mathrm{P}=$ paretic/nondominant side percentage load distribution.

Abbreviations: Al, asymmetry index; GGl, Gillette Gait Index; IC, initial contact; ROM, range of motion. 
ratios (SRs). ${ }^{16,29}$ Since perfect symmetry is represented by an SR value of 0 , the results could be easily interpreted to facilitate comparisons between studies. The mean AI value obtained in the current study (average AI $>0.09$ ) was slightly lower than that in other studies. ${ }^{7-9}$ This finding may be explained by the fact that this study included individuals with chronic stroke (average time PS $<2$ years; Table 1).

Our experiments revealed significant differences in gait patterns between hemiplegic patients and healthy individuals in terms of both spatiotemporal and kinematic parameters. The spatiotemporal differences detected were greater step length asymmetry and reduced walking speed of PS gait (Table 1), while kinematic differences included disorders in $3 \mathrm{D}$ pelvic movement and motion of the hip in the sagittal and coronal planes as well as knee and ankle dorsiflexion in both gait phases (Table 2). In addition, hemiparetic patients exhibited large interindividual variabilities in joint kinematics (Table 2). Data from the current study support the consensus that PS gait is usually characterized by spatiotemporal abnormalities mainly resulting from step length asymmetry, such as asymmetry of stance time, single stance time, increased double support phase, and swing time. ${ }^{14-17}$

The joint kinematic deviations of the paretic and/or nonparetic lower limb during walking after stroke relative to healthy subjects include distinct joint (hip, knee, and ankle) movements and are analyzed separately for stance and swing phases. The most commonly reported kinematic deviations of PS gait are asymmetrical movement of pelvis, insufficient paretic hip and knee flexion in swing phase, and excessive ankle plantar flexion. ${ }^{18,19,29,32}$

While a number of spatiotemporal and kinematic abnormalities of hemiparetic gait have been reported in the past, recent research has focused on identifying a single indicator for effectively describing PS gait abnormalities. In this case, quantitative 3DGA and summary scores, such as GGI, provide the best means to evaluate complex multifactorial gait dysfunction in hemiparetic patients. Given that GGI describes a deviation relative to normal set parameters, the measurement allows a general assessment of gait pathology of the examined individuals, compared with healthy controls. In terms of the GGI values determined in our study, two facts deserve attention: 1) GGI values of paretic limbs, on average, were nearly twofold greater than those of nonparetic limbs; and 2) the degree of gait deviation was significantly greater in PS, compared with healthy controls (Table 1).

As reported by Schutte et al and Gage, more severe diagnoses, on average, would correspond to greater gait abnormalities, resulting in higher index scores. ${ }^{23,33} \mathrm{GGI}$, which is commonly applied as a valuable tool for assessing gait in children with cerebral palsy, ${ }^{23,33,34}$ may also be effectively used to evaluate gait abnormalities in adults with CNS disorders. ${ }^{24,35}$ Although Krawczyk et al $^{35}$ reported higher GGI $(\sim 140)$ than that in our experiments, data from both studies validated the efficiency of this index in categorizing gait pathology and its utility as an indicator of the degree of PS gait deviation. It is important to note that the population examined in the previous study was considerably more heterogeneous and included individuals with hemorrhagic stroke as well as those in subacute stages following stroke, which may underlie the observed differences in gait pathology.

The current results are consistent with previous findings on reduced WB of the paretic limb in standing and asymmetry of PS gait, supporting our hypothesis on the relationship between WBA of quiet standing and gait deviation in individuals with chronic stroke. As shown in our study, the degree of WBA in standing (percentage decrease in WB on the paretic lower limb) was strongly positively related to deviation from normal gait, evaluated based on GGI.

Exploration of the correlations between WBA and 16 distinct gait parameters constituting GGI for the paretic lower limbs should aid in further clarifying potential associations between standing asymmetry and a few specific spatiotemporal and kinematic indicators of gait abnormalities. In particular, the result that increased WB on the nonparetic limb in quiet standing was associated with increased asymmetry of paretic step length and decreased walking speed (Table 3) was interesting. The relationship between WBA and spatial and temporal gait parameters continues to raise many interesting questions for further research. ${ }^{13-17} \mathrm{~A}$ few studies have explored the associations of weight shift asymmetry in relation to gait spatiotemporal deviations in the PS population. Lewek et $\mathrm{al}^{21}$ demonstrated that the weight distribution between paretic and nonparetic lower limbs during quiet standing is negatively correlated with stance time asymmetry in fast walking. Hendrickson et al further reported that increased WB on the nonparetic limb in quiet standing and reduced capacity to bear weight on the paretic limb are related to increased asymmetry of swing time, stance time, and, to a lesser extent, step length. ${ }^{20}$ Here, we additionally revealed the relationships between WBA and kinematic parameters constituting GGI. The main finding was that WBA with increased WB on the nonparetic limb in quiet standing is related to impairment of paretic knee and ankle kinematics in the sagittal plane, such as insufficient ROM of knee flexion in the gait cycle and deficit in ankle dorsiflexion in the swing phase. 
Although relationships of WBA with standing balance control, ${ }^{5-7,9}$ spatiotemporal gait parameters, ${ }^{13,14,17,36}$ sensorimotor deficits, ${ }^{2,9,10}$ biomechanical impairments, ${ }^{3,4,13,14,37}$ and even falls have been previously reported, no clear evidence showing associations between WBA in standing and kinematics deviations of PS gait is available. To our knowledge, this study is the first to examine the relationship between WB on the paretic limb in quiet standing and GGI and kinematic parameters constituting GGI of hemiplegic gait. The results validate the theory that impairment in symmetry of WB between paretic and nonparetic lower limbs contributes to kinematic knee and ankle deviations in PS gait. Recent studies have additionally suggested that the levels of WBA and different gait parameters could be more relevant than walking speed in determining the degree of paretic leg impairment in PS individuals during locomotion. . $^{16,17,29}$

A number of limitations need to be taken into account when interpreting our results. First, the findings from treadmill-based gait research can differ from overground gait data. Second, the present study included a relatively small number of participants, and the included population was a convenience sample (individuals with chronic stroke) and not based on specific sample size calculations. Nevertheless, our preliminary findings are significant and pave the way for further studies with larger sample sizes, taking into account the different degrees of severity (mild, moderate, severe, and very severe) and characteristics of stroke.

\section{Summary and conclusion}

Overall, WBA and decreased contribution of the paretic limb in quiet standing were associated with increased PS gait deviation from normal. Our findings indicate that the significant associations of WBA with gait deviation and spatiotemporal and kinematic abnormalities are due to a common link with lower limb motor impairment. Further research is needed to determine the causal link between asymmetric WB and gait abnormalities and to identify the specific determinants of PS gait asymmetry.

Asymmetrical standing posture and asymmetrical gait PS, although major targets in stroke rehabilitation, remain poorly understood at present. ${ }^{38}$ The results of this study are potentially clinically relevant and confirm the importance of weight shift symmetry in standing and walking, leading to the viewpoint that WBA between paretic and nonparetic body sides should be a primary focus of early assessment after stroke.

In view of the collective data, we propose that specific early treatment approaches based on 1) the inhibition of weight shift to the nonparetic side and 2) the facilitation of symmetrical weight distribution between body sides in any position (supine, prone, sitting, kneeling, and standing) in acute stroke patients in the early stages of rehabilitation should aid in improving gait symmetry.

\section{Author contributions}

AS and MDS contributed to the conception and design of the study, performed the experiments, and interpreted the data. AZ analyzed the data. MDS and AS wrote the article. ALB made critical revisions to the manuscript. All authors contributed toward data analysis, drafting, and critically revising the paper and agreed to be accountable for all aspects of the work.

\section{Disclosure}

The authors report no conflicts of interest in this work.

\section{References}

1. Rothwell PM, Coull AJ, Silver LE, et al. Population-based study of event-rate, incidence, case fatality, and mortality for all acute vascular events in all arterial territories (Oxford Vascular Study). Lancet. 2005; 366(9499):1773-1783.

2. Marigold DS, Eng JJ. The relationship of asymmetric weight-bearing with postural sway and visual reliance in stroke. Gait Posture. 2006;23(2): 249-255.

3. De Bujanda E, Nadeau S, Bourbonnais D. Pelvic and shoulder movements in the frontal plane during treadmill walking in adults with stroke. J Stroke Cerebrovasc Dis. 2004;13(2):58-69.

4. Olney SJ, Griffin MP, McBride ID. Multivariate examination of data from gait analysis of persons with stroke. Phys Ther. 1998;78(8):814-828.

5. Laufer Y, Sivan D, Schwarzmann R, Sprecher E. Standing balance and functional recovery of patients with right and left hemiparesis in the early stages of rehabilitation. Neurorehabil Neural Repair. 2003; 17(4):207-213.

6. Yanohara R, Teranishi T, Tomita Y, Tanino G, Ueno Y, Sonoda S Recovery process of standing postural control in hemiplegia after stroke. J Phys Ther Sci. 2014;26(11):1761-1765.

7. Sheikh M, Azarpazhooh MR, Hosseini HA. The effect of immediate decreasing of weight bearing asymmetry on quiet standing postural control in individuals with chronic stroke. Physiother Theory Pract. 2017;33(10):751-757.

8. Pérennou D. Weight bearing asymmetry in standing hemiparetic patients. J Neurol Neurosurg Psychiatry. 2005;76(5):621.

9. Brière A, Lauzière $S$, Gravel D, Nadeau S. Perception of weight-bearing distribution during sit-to-stand tasks in hemiparetic and healthy individuals. Stroke. 2010;41(8):1704-1708.

10. Pérennou D, Bronstein A. Balance disorders and vertigo after stroke: assessment and rehabilitation. In: Bogousslavsky J, Barnes M, Dobkin B, editors. Recovery After Stroke. Cambridge: Cambridge University Press; 2005:320-398.

11. De Quervain IA, Simon SR, Leurgans S, Pease WS, McAllister D. Gait pattern in early recovery period after stroke. J Bone Joint Surg Am. 1996;78(10):1506-1514.

12. Viosca E, Lafuente R, Martínez JL, Almagro PL, Gracia A, González C. Walking recovery after an acute stroke: assessment with a new functional classification and the Barthel Index. Arch Phys Med Rehabil. 2005; 86(6):1239-1244.

13. Bohannon RW, Andrews AW. Relationship between impairments and gait performance after stroke: a summary of relevant research. Gait Posture. 1995;3(4):236-240. 
14. Chen G, Patten C, Kothari DH, Zajac FE. Gait differences between individuals with post-stroke hemiparesis and non-disabled controls at matched speeds. Gait Posture. 2005;22(1):51-56.

15. Patterson KK, Parafianowicz I, Danells CJ, et al. Gait asymmetry in community-ambulating stroke survivors. Arch Phys Med Rehabil. 2008; 89(2):304-310.

16. Patterson KK, Gage WH, Brooks D, Black SE, McIlroy WE. Evaluation of gait symmetry after stroke: a comparison of current methods and recommendations for standardization. Gait Posture. 2010;31(2): 241-246.

17. Patterson KK, Gage WH, Brooks D, Black SE, McIlroy WE. Changes in gait symmetry and velocity after stroke: a cross-sectional study from weeks to years after stroke. Neurorehabil Neural Repair. 2010; 24(9):783-790.

18. Hsu AL, Tang PF, Jan MH. Analysis of impairments influencing gait velocity and asymmetry of hemiplegic patients after mild to moderate stroke. Arch Phys Med Rehabil. 2003;84(8):1185-1193.

19. Balaban B, Tok F. Gait disturbances in patients with stroke. PMR. 2014;6(7):635-642.

20. Hendrickson J, Patterson KK, Inness EL, McIlroy WE, Mansfield A. Relationship between asymmetry of quiet standing balance control and walking post-stroke. Gait Posture. 2014;39(1):177-181.

21. Lewek MD, Bradley CE, Wutzke CJ, Zinder SM. The relationship between spatiotemporal gait asymmetry and balance in individuals with chronic stroke. J Appl Biomech. 2014;30(1):31-36.

22. Nardone A, Godi M, Grasso M, Guglielmetti S, Schieppati M. Stabilometry is a predictor of gait performance in chronic hemiparetic stroke patients. Gait Posture. 2009;30(1):5-10.

23. Schutte LM, Narayanan U, Stout JL, Selber P, Gage JR, Schwartz MH. An index for quantifying deviations from normal gait. Gait Posture. 2000;11(1):25-31.

24. Cretual A, Bervet K, Ballaz L. Gillette Gait Index in adults. Gait Posture. 2010;32(3):307-310.

25. Brunnström S. Movement Therapy in Hemiplegia: A Neuro-physiological Approach. New York: Harper \& Row; 1970.

26. Ansari NN, Naghdi S, Moammeri H, Jalaie S. Ashworth scales are unreliable for the assessment of muscle spasticity. Physiother Theory Pract. 2006;22(3):119-125.
27. Paternostro-Sluga T, Grim-Stieger M, Posch M, et al. Reliability and validity of the Medical Research Council (MRC) scale and a modified scale for testing muscle strength in patients with radial palsy. $J$ Rehabil Med. 2008;40(8):665-671.

28. Altman DG. Practical Statistics for Medical Research. London, UK: Chapman and Hall/CRC; 1991.

29. Lauzière S, Betschart M, Aissaoui R, Nadeau S. Understanding spatial and temporal gait asymmetries in individuals post stroke. Int J Phys Med Rehabil. 2014;2(3):201.

30. Genthon N, Rougier P, Gissot AS, Froger J, Pélissier J, Pérennou D. Contribution of each lower limb to upright standing in stroke patients. Stroke. 2008;39(6):1793-1799.

31. Rougier PR, Genthon N. Dynamical assessment of weight-bearing asymmetry during upright quiet stance in humans. Gait Posture. 2009;29(3): 437-443.

32. Beyaert C, Vasa R, Frykberg GE. Gait post-stroke: pathophysiology and rehabilitation strategies. Neurophysiol Clin. 2015;45(4-5):335-355.

33. Gage JR, Schwartz MH, Koop SE, Novacheck TF, editors. The identification and treatment of gait problems in cerebral palsy. In: Clinics in Developmental Medicine. London: Mac Keith Press; 2009.

34. Szopa A, Domagalska-Szopa M, Czamara A. Gait pattern differences in children with unilateral cerebral palsy. Res Dev Disabil. 2014;35: 2261-2266.

35. Krawczyk M, Szczerbik E, Syczewska M. The comparison of two physiotherapeutic approaches for gait improvement in sub-acute stroke patients. Acta Bioeng Biomech. 2014;16(1):11-18.

36. Balasubramanian CK, Bowden MG, Neptune RR, Kautz SA. Relationship between step length asymmetry and walking performance in subjects with chronic hemiparesis. Arch Phys Med Rehabil. 2007;88: 43-49.

37. Syczewska M, Öberg T. Spinal segmental movement changes during treadmill gait after stroke. J Hum Kinetics. 2006;16:39-56.

38. Patterson KK, Mansfield A, Biasin L, Brunton K, Inness EL, McIlroy WE. Longitudinal changes in poststroke spatiotemporal gait asymmetry over inpatient rehabilitation. Neurorehabil Neural Repair. 2015;29(2): $153-162$.
Clinical Interventions in Aging

\section{Publish your work in this journal}

Clinical Interventions in Aging is an international, peer-reviewed journal focusing on evidence-based reports on the value or lack thereof of treatments intended to prevent or delay the onset of maladaptive correlates of aging in human beings. This journal is indexed on PubMed Central, MedLine,

\section{Dovepress}

CAS, Scopus and the Elsevier Bibliographic databases. The manuscript management system is completely online and includes a very quick and fair peer-review system, which is all easy to use. Visit http://www.dovepress. com/testimonials.php to read real quotes from published authors. 